\title{
A screen of shRNAs targeting tumor suppressor genes to identify factors involved in A549 paclitaxel sensitivity
}

\author{
DIANA JI, STACY L. DEEDS and EDWARD J. WEINSTEIN
}

\author{
Sigma-Aldrich, Functional Genomics Operations, 2909 Laclede Ave., St. Louis, MO 63103, USA
}

Received March 2, 2007; Accepted June 13, 2007

\begin{abstract}
An shRNA tumor suppressor panel was screened using reverse infection of an A549 tumorigenic cell line and exposing it to a predetermined concentration of paclitaxel, an anticancer drug. The shRNAs targeting a positive control gene, $M D R 1$, were found to effectively decrease mRNA levels and cause cells to become more sensitive to the chemotherapeutic drug. A set of genes were identified in the screen of a panel of tumor suppressors which, when down-regulated, were found to increase or decrease cell sensitivity in regards to treatment with paclitaxel. In many cases, there were multiple clones to a single gene that provided a positive result. The shRNAs targeting SMAD4, LZTS2, ST14 and VHL all increased the cell's sensitivity to paclitaxel. The loss of other tumor suppressors such as GLTSCR2, LATS1, NF1, PTEN, TP53 and WT1 induced a protective effect in the cell, making it more resistant to the effect of the drug. Further investigation of VHL mRNA levels after down-regulation with shRNA show a direct correlation between gene expression levels and paclitaxel sensitivity. This study credits the identified genes with the potential to act as prognostic biomarkers for use in genetic profiling, or even as targets in pathways of tumorigenesis yet to be fully understood.
\end{abstract}

\section{Introduction}

Lung cancer is a leading cause of death in the United States, with an estimated number of over 172,000 new cases and 163,000 deaths in 2005. Lung cancer accounts for almost $30 \%$ of all cancer related deaths in the United States (1). The incidence of lung cancer has declined in recent years, largely due to a decrease in smoking rates. Yet lung cancer is a serious disease, with a low 5-year survival rate and very few patients receiving effective chemotherapy (reviewed in 2).

Paclitaxel, produced by Bristol-Meyers Oncology, is an antimicrotubule agent that works by stabilizing tubulin

Correspondence to: Dr Edward J. Weinstein, Functional Genomics, Sigma-Aldrich, 2909 Laclede Ave., St. Louis, MO 63103, USA

E-mail: eweinstein@sial.com

Key words: shRNA, lentivirus, tumor suppressor, paclitaxel, chemosensitivity, biomarkers, RNAi polymer formation and shifting the equilibrium in cancer cells towards microtubule assembly. The normal function of the microtubule network is thus disrupted, resulting in abnormal mitotic processes. Early treatment regimens examining the effectiveness of paclitaxel in non-small cell lung cancer (NSCLC) showed response rates of 21 to $24 \%(3,4)$ and an improved one-year survival rate, approaching $40 \%$ of patients treated. Due to the initial success of this chemotherapy, several combination trials have been executed, or are in the process, in the hope of finding paclitaxel combinations with increased efficacy, including paclitaxel combined with cisplatin (5), paclitaxel with carboplatin (reviewed in 6) and paclitaxel with radiation therapy (7).

One way to improve the response rates to chemotherapies such as paclitaxel, other than by a combination with multiple therapies, is to predict the chemosensitivity of individual patients to therapy. This requires, in part, an understanding of the individual genes involved in therapy response. It also requires an effective and integrated use of a variety of genomic-based tools. Over the past few years, significant use has been made of genome-wide expression profile analyses and the correlation of microarray data with clinical response to therapies. This has been most evident and successful in the field of breast cancer research $(8,9)$.

The use of RNA interference technology (RNAi) now allows for genome-wide screening of molecular alterations in a variety of cell lines. The broad use of siRNAs has shown the advantages of knocking down individual transcripts in easily transfected and readily assayed cell lines (10). The use of shRNA libraries for genetic screens has demonstrated an advantage of shRNA over siRNA in that difficult-to-transfect cells can be utilized (often allowing for a more realistic phenotype in a more relevant cell type) and longer-term assays can be performed (due to the stable knockdown potential of shRNA). While others have elucidated potential factors in attenuation of sensitivity of cells to therapy through the use of siRNA screens (11) we have utilized a lentiviral-based shRNA library screen to identify sensitizing factors in the use of paclitaxel in NSCLC.

\section{Materials and methods}

Cell culture. The human lung adenocarcinoma cell line A549 (ATCC) was cultured in Ham's F12 medium supplemented with $10 \% \mathrm{v} / \mathrm{v}$ fetal bovine serum, $4 \mathrm{mM}$ final L-glutamine and penicillin/streptomycin (all reagents obtained from SigmaAldrich unless otherwise noted, St. Louis, MO) in $T 75 \mathrm{~cm}^{2}$ 
cell culture flasks, at $37^{\circ} \mathrm{C}$ and $5 \% \mathrm{CO}_{2}$. At $\sim 80 \%$ confluence, the cells were trypsinized and reseeded into 96 -well plates for transduction.

Plasmid Midiprep. Isolation of the plasmid DNA from MDR1 constructs was performed using GenElute HP Plasmid Midiprep kit (Sigma-Aldrich) following the standard protocol as found in the enclosed technical bulletin. The shRNA DNA plasmids used are: TRCN0000059683 (MDR1-1), TRCN0000059684 (MDR1-2), TRCN0000059685 (MDR1-3), TRCN0000059686 (MDR1-4) and TRCN0000059687 (MDR1-5) (Sigma-Aldrich). DNA was normalized to $20 \mu \mathrm{g} / \mathrm{ml}$ in a 1:10 dilution of DNA and TE, and analyzed using SoftMaxPro computer software. For the screen, DNA was prepared in 96-well format (Invitrogen, Carlsbad, CA).

Virus production. Transfections were performed in a sub-strain of HEK293T cells (ATCC, Manassas, VA) and virus particles were harvested for 40 and $48 \mathrm{~h}$ post-transfection, yielding $\sim 200 \mu 1$ per sample. A lipid-based transfection reagent was used for lentiviral production with packaging construct (pDelta 8.9), envelope construct (pCMV-VSV-G) and five independent shRNA constructs in serum free DME media for all transfections, according to the manufacturer's instructions. The viral titer was determined by p24 ELISA assay (Zeptometrix, Buffalo, NY) according to the manufacturer's instructions.

Transduction of MDR1. The human lung adenocarcinoma cell line, A549, was infected at a MOI of $\sim 10$. There were 40,000 cells per well seeded in a 24 -well plate. Cells were allowed $24 \mathrm{~h}$ to attach and were infected by the addition of virus, plus hexadimethrine bromide (Sigma-Aldrich) at a final concentration of $8 \mu \mathrm{g} / \mathrm{ml}$. Empty vector virus (pLKO.1, catalog number SHC001V, Sigma-Aldrich) acted as a positive control for puromycin selection as well as a negative control for MDRl knockdown. Four replicates of each construct were infected and duplicates were made of the control and blank wells. Cells were selected with $2 \mu \mathrm{g} / \mathrm{ml}$ puromycin for $48 \mathrm{~h}$ post-infection. Morphological changes were observed and photographed with an inverted phase contrast microscope after each succeeding event.

Quantitative real-time PCR. Transduced cells were harvested after five days of selection with puromycin and RNA was isolated using GenElute Mammalian Total RNA Kit (SigmaAldrich). TaqMan Gene Expression Assay primer and probe sets were used for target amplification and detection (Applied Biosystems, Foster City, CA). MDRl primers were 5'- labeled with FAM reporter dye and 3'-labeled with fluorescent quencher. QRT-PCR was preformed using a master mix kit prepared with Sigma Quantitative RT-PCR ReadyMix (QR200) supplemented with $\mathrm{MgCl}_{2}$ and water. Reference dye was also included as an internal control for fluorescence. Reactions of $20 \mu 1$ total volume were set up, using $4 \mu 1$ of RNA. All reactions were run and analyzed with the MX3000 qPCR system and software (Stratagene, La Jolla, CA). Cycling parameters were as follows: $15 \mathrm{~min}$ at $42^{\circ} \mathrm{C}$, $3 \mathrm{~min}$ at $94^{\circ} \mathrm{C}$ and 45 cycles of $15 \mathrm{sec}$ at $94^{\circ} \mathrm{C}$ and $1 \mathrm{~min}$ at $60^{\circ} \mathrm{C}$. Expression of MDRl from the stably infected cell lines was compared against GAPDH mRNA for quantification. Assays were performed in triplicate and values are expressed with pLKO.1 expression normalized to $100 \%$.

Cytotoxicity assays. In vitro cytotoxicity assays were performed using the Quick Cell Proliferation Assay Kit (BioVision, Mountain View, CA). A549 cells were plated overnight in 96-well plates at a density of 40,000 cells $/ \mathrm{cm}^{2}$. Increasing concentrations of paclitaxel (Sigma-Aldrich) in Ham's F12 media replaced the normal media. After $24 \mathrm{~h}$ of culture with the chemotherapeutic drug, $10 \mu 1$ of WST- 1 was added to each well and the plates were incubated for an additional $4 \mathrm{~h}$. The formazan dye produced by live cells was then read by a spectrophotometer for absorbance at $450 \mathrm{~nm}$. Absorbance measurements were normalized by subtracting the value of blank wells from the wells with cultured cells.

Reverse infection screen. Reverse infection was performed by pre-plating $5 \mu \mathrm{l}$ of virus from a tumor suppressor panel and adding A549 cells at a seeding density of 16,000 cells/ well in 96-well plates. Triplicates of pLKO.1 virus were included as a negative control and MDR1-2 (TRCN0000059684) virus as a positive control. Final concentration of polybrene at $8 \mu \mathrm{g} / \mathrm{ml}$ was added and $3 \mu \mathrm{g} / \mathrm{ml}$ of puromycin was used to select at $48 \mathrm{~h}$ post-infection.

QuantiGene measurement of mRNA. Five separate A549 cell lines were established, each containing an shRNA targeting VHL and a negative control, pLKO.1. Lysate was harvested and gene expression measured according to the manufacturer's instructions for QuantiGene Reagent System (Panomics, Fremont, CA).

\section{Results}

Prior to screening a panel of shRNAs, a positive control was investigated to establish that A549 cells could have their level of paclitaxel sensitivity altered by genetic modification. DNA plasmids to five shRNAs targeting the drug pump MDRl were prepared which had been cloned into the pLKO.1 vector and therefore suitable for lentivirus production. All five shRNAs target the coding region of the gene and were designed by a publicly available algorithm provided by the RNAi Consortium (Table I). MDRl was chosen as a positive control due to its well-documented physiological role as a drug pump (12).

Lentiviral particles with the vsv-g coat protein were produced for each shRNA targeting $M D R l$ and titering units were measured by a p24 assay. A549 cells were seeded into a 24-well plate and infected with the virus at MOI of $\sim 10$. After incubation with the virus for $24 \mathrm{~h}$, fresh media containing puromycin for selection was applied to the cells. Six stable cell lines were then established - one for each shRNA MDRl clone infection and one for the empty virus negative control infection, pLKO.1.

RNA was isolated from each of the established cell lines and the level of MDRl was then measured by quantitative RT-PCR. Target gene levels were then normalized to the housekeeping gene GAPDH in order to allow for comparison between cell lines (Fig. 1). Three of the five shRNAs resulted 
Table I. shRNA sequences targeting MDRl (NM_000927).

\begin{tabular}{lcc}
\hline Sequence & $\begin{array}{c}\text { RNAi consortium } \\
\text { number }\end{array}$ & Region targeted \\
\hline CCGGCCGAACACATTGGAAGGAAATCTCGAGATTTCCTTCCAATGTGTTCGGTTTTTG & TRCN0000059683 & CCGAACACATTGGAAGGAAAT \\
CCGGGCAGCAATTAGAACTGTGATTCTCGAGAATCACAGTTCTAATTGCTGCTTTTTG & TRCN0000059684 & GCAGCAATTAGAACTGTGATT \\
CCGGCGACAGAATAGTAACTTGTTTCTCGAGAAACAAGTTACTATTCTGTCGTTTTTGC & TRCN0000059685 & CGACAGAATAGTAACTTGTTT \\
CCGGGCTCATCGTTTGTCTACAGTTCTCGAGAACTGTAGACAAACGATGAGCTTTTTGC & TRCN0000059686 & GCTCATCGTTTGTCTACAGTT \\
CCGGGCTGCTTTCCTGCTGATCTATCTCGAGATAGATCAGCAGGAAAGCAGCTTTTTGC & TRCN0000059687 & GCTGCTTTCCTGCTGATCTAT \\
\hline
\end{tabular}

Algorithm design rules can be found at www.broad.mit.edu/genome_bio/trc/rules.html.

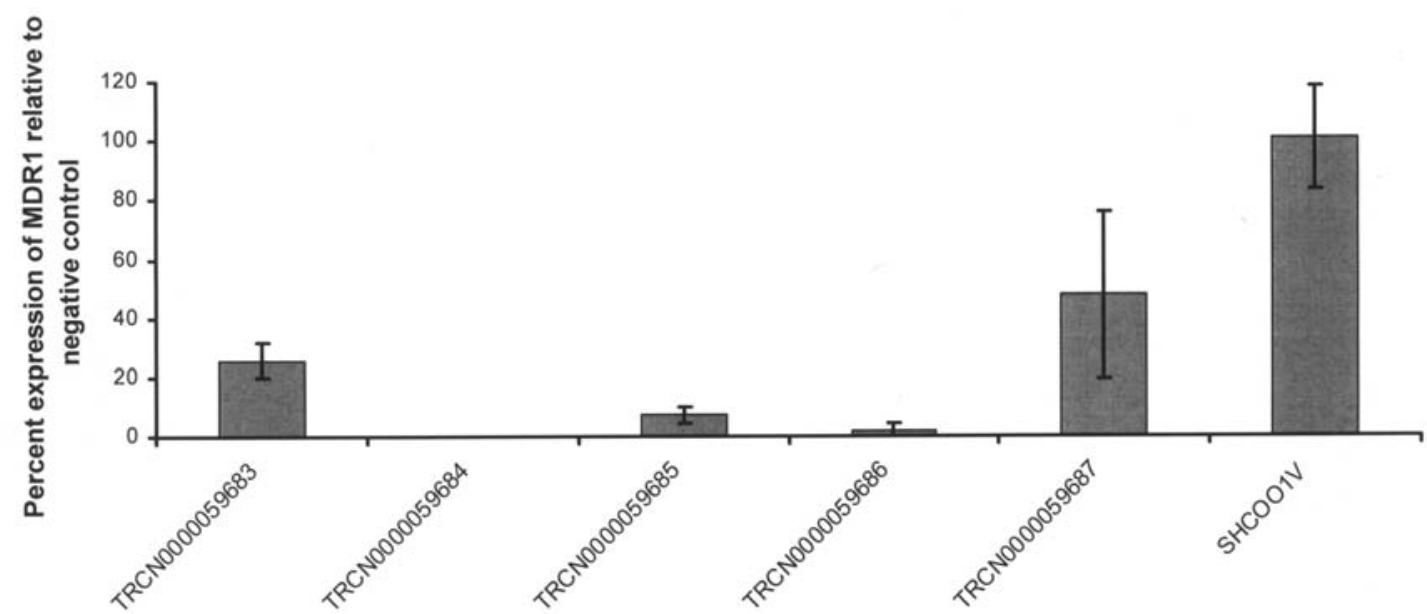

MDR1 gene shRNA infected cells

Figure 1. Quantitative RT-PCR measurements of cell lines infected with shRNA targeting MDRl and a negative control. All measurements were normalized to GAPDH.

in MDRl transcript knockdowns of $>90 \%$ (TRCN0000059684, TRCN0000059685 and TRCN0000059686) with one of those three resulting in gene knockdown below the level of detection (TRCN0000059684). One shRNA gave a partial knockdown of $\sim 75 \%$ (TRCN0000059683) while the last one resulted in only a $50 \%$ decrease in endogenous mRNA (TRCN0000059687). This varying level of efficacy by multiple shRNAs derived from a common algorithm is typical and demonstrates the importance of validating individual lentiviral constructs.

In order to determine if a variation in MDR1 levels would have an effect on A549 cell sensitivity to paclitaxel, each cell line was treated with multiple concentrations of paclitaxel ranging from 1 to $10 \mu \mathrm{M}$. After $24 \mathrm{~h}$ in culture in the presence of the chemotherapeutic agent, cell survival was measured (Fig. 2). While the parental A549 cell line displayed the most resistance to paclitaxel, the cell line with the greatest knockdown of MDR1, A549/sh59684, displayed the greatest sensitivity to the drug at $5 \mu \mathrm{M}$. Another MDR1 shRNA infected cell line, A549/sh59686, with over 95\% target transcript knockdown, also showed significant increased sensitivity to paclitaxel compared to its parental cell line.

With a clear correlation established between gene knockdown by shRNA and cell sensitivity to paclitaxel, a panel of shRNAs were next screened in order to identify paclitaxel sensitizing and desensitizing genes. Lentiviral particles from a tumor suppressor panel were utilized via 'reverse infection' of A549 cells. The panel consisted of shRNAs targetting 74 different genes, all identified as tumor suppressors. Five microliters of each tumor suppressor lentiviral clone, each encoding a unique shRNA, were aliquoted into 96-well tissue culture plates. The A549 cells were then added to the wells at a seeding density of 16,000 cells per well and allowed $24 \mathrm{~h}$ for infection. Following selection with puromycin, cells were split 1:2, allowed another 2 days to reach $80 \%$ confluence and either treated with $5 \mu \mathrm{M}$ paclitaxel or mock-treated. Cells, both treated and untreated, were then measured for survival and a comparison was made for each shRNA transduced (Fig. 3). The ratio for cell survival was calculated between negative-control (Sigma, catalog \#SHC001V) infected cells that were either paclitaxel treated or mock-treated. This ratio was then set to equal 1 and utilized to normalize the ratio of survival for cells within each shRNA infection.

Several shRNAs that led to a knockdown of tumor suppressor genes resulted in decreased sensitivity to paclitaxel. Six instances were found where multiple clones to the same gene resulted in the most significant increase in resistance to the drug when knocked out. There were three shRNA clones targeting PTEN and two shRNA clones each targeting TP53, 


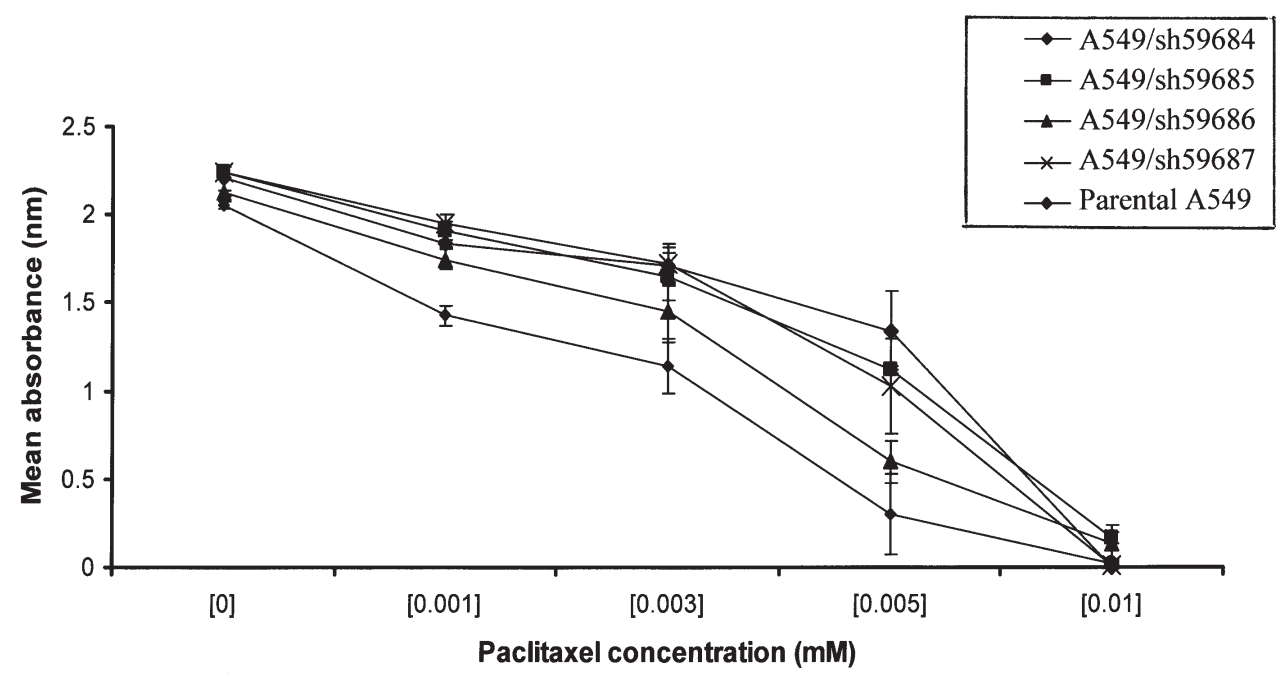

Figure 2. Cell death from increasing concentrations of paclitaxel on MDR1 shRNA infected A549 cells. Cells were treated with various concentrations of paclitaxel for $24 \mathrm{~h}$ and assayed with WST-1 reagent. All measurements were performed in triplicate.

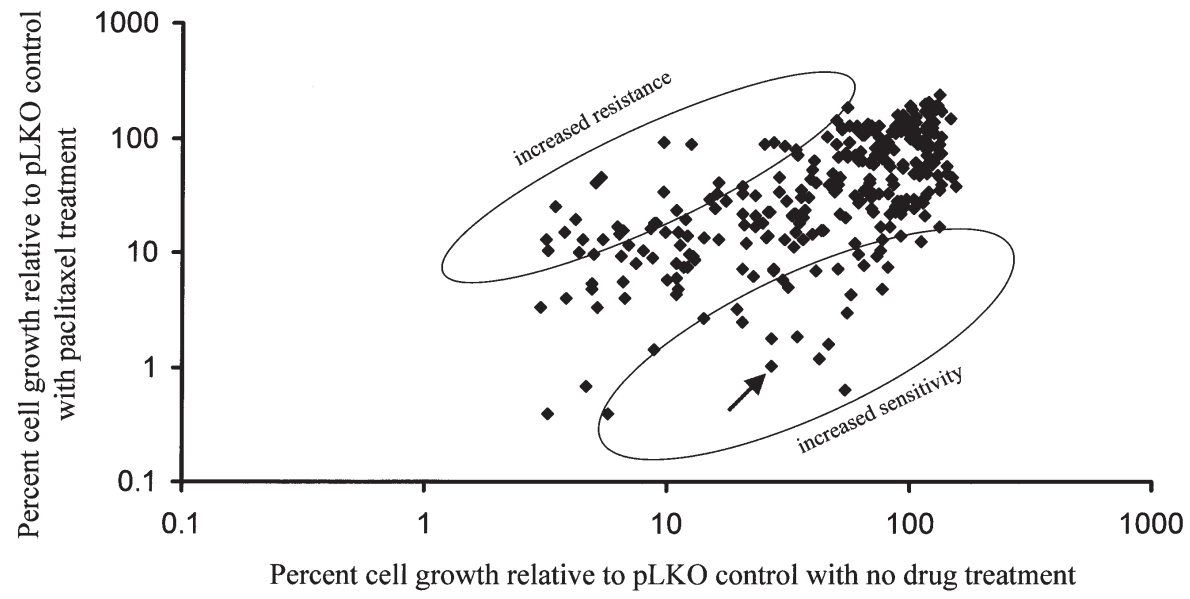

Figure 3. Screen of a tumor suppressor panel containing 337 shRNAs targeting 74 genes. After mock-treatment, or treatment with paclitaxel, cell survival was measured and normalized to cell survival for cells infected with negative-control shRNA. Data are plotted on each axis by percent survival. The arrow notes cell growth for cells infected with the positive control for sensitivity, shRNA to MDR1.

GLTSCR2, LATS1,NF1 and WT1, all resulting in an increased resistance ratio (cell survival with shRNA alone to cell survival with shRNA plus paclitaxel) between 2.3 -fold and 8.6-fold (Fig. 4a). These results are in line with previously published results showing a link between the loss of a tumor suppressor gene and an increased resistance to chemotherapy (13).

In examining which shRNAs, when infected into A549 cells, result in the most paclitaxel sensitization, four genes were identified that were targeted by 15 clones (Fig. 4b). Three shRNAs targeting VHL resulted in an increased sensitivity ratio (cell survival with shRNA alone to cell survival with shRNA plus paclitaxel). Similarly, 5 clones of SMAD4, 4 clones of ST14 and 3 clones of LZTS2 all resulted in increased sensitivity by at least $80 \%$. While it may seem counterintuitive to link decreased levels of tumor suppressor genes to increased levels of sensitivity, these results are reflected in the literature, as in the case with cisplatin and Brca1 $(14,15)$.

Next, one of the target genes, VHL (von Hippel-Lindau), was chosen to further validate its role in drug sensitivity. Four separate stable cell lines were established from parental A549 cells, each containing a different shRNA clone targeting $V H L$ (TRCN0000039623, TRCN0000039624, TRCN0000039625, and TRCN0000039626). A control line was also established with the stable infection of the empty control plasmid (pLKO.1). The levels of VHL expression remaining in the cells were measured using the QuantiGene reagent system. When the results were compared to the data obtained from the screen, there was a strong correlation between levels of gene expressed and degrees of sensitivity to paclitaxel (Fig. 5). The three shRNA clones targetting VHL that resulted in a stable knockdown of $\sim 75$ to $85 \%$ of the endogenous levels also resulted in increased paclitaxel sensitivity. The single clone that decreased the endogenous $V H L$ level by only $60 \%$ failed to sensitize cells to paclitaxel.

\section{Discussion}

Every tumor is genetically heterogeneous; their molecular makeup helps determine if a patient will respond positively to 


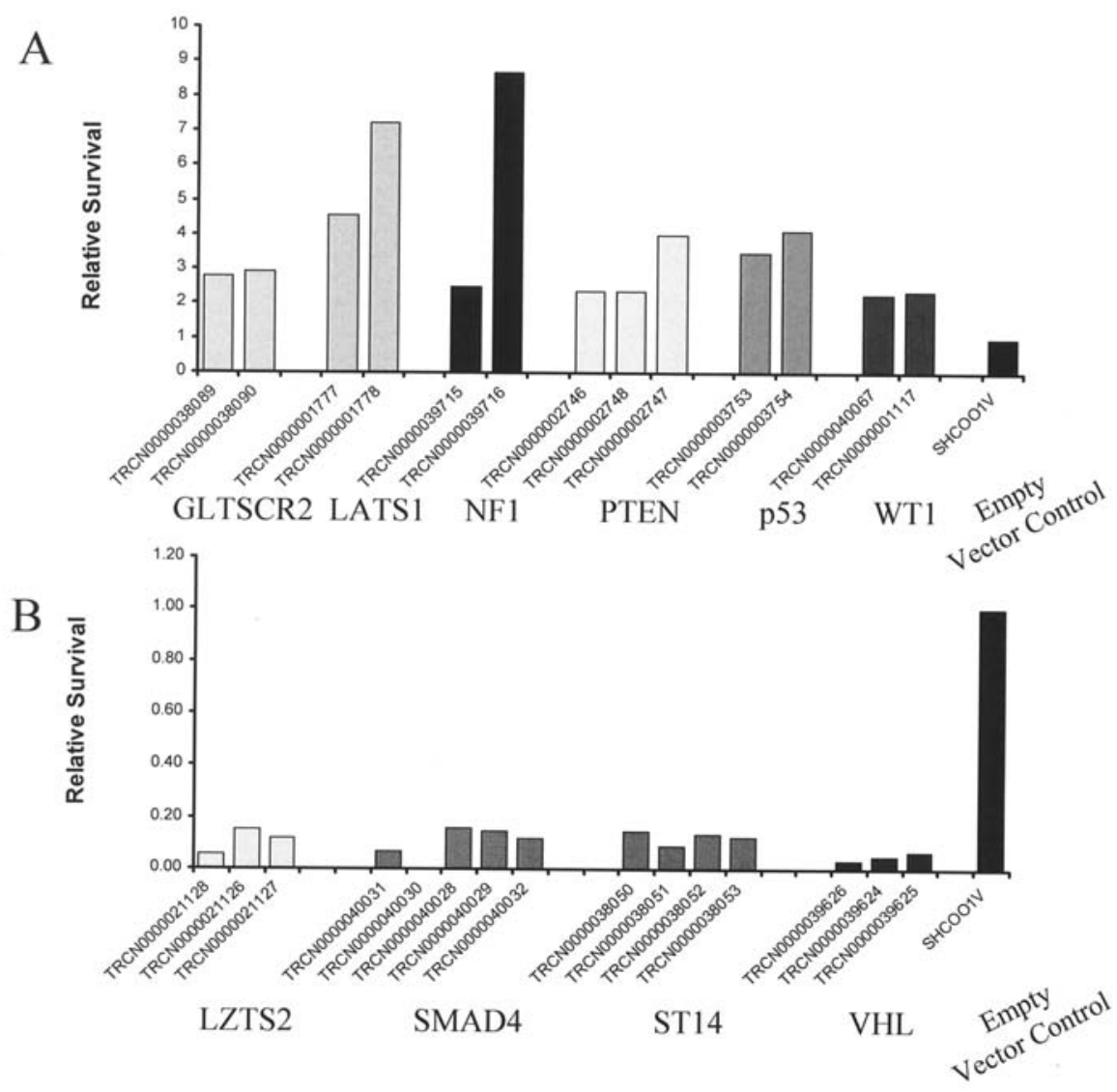

Figure 4. Gene knockdown resulting in the most enhanced sensitivity or resistance to paclitaxel. Relative knockdown is shown for the most significant cases where more than one shRNA to a target gene results in (A) increased resistance to paclitaxel or (B) increased sensitivity. All values are relative to the ratio for treated: untreated cells infected with the negative control shRNA.

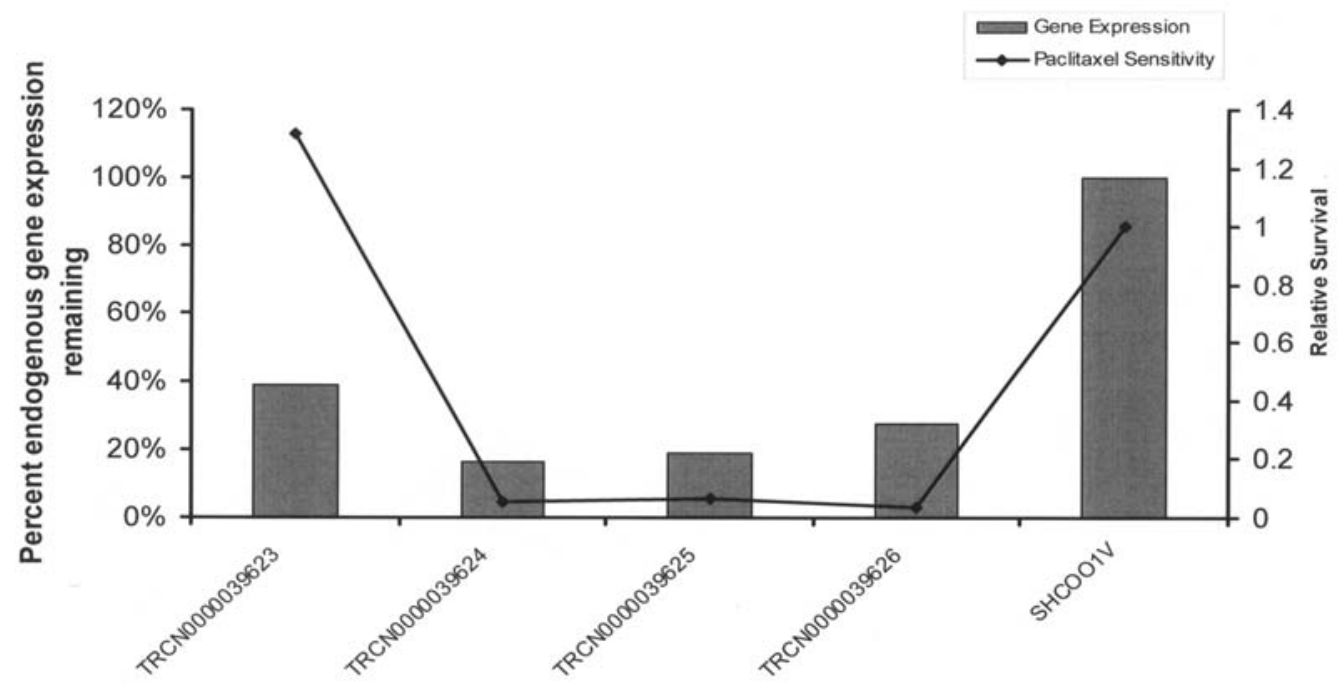

VHL gene shRNA infected cells

Figure 5. Direct correlation between $V H L$ gene expression and cell's sensitivity to paclitaxel. A549 cell lines infected with shRNAs targeting $V H L$ and a negative control are measured for gene expression levels. The amount of $V H L$ remaining in the cell lines has a direct correlation to its sensitivity to paclitaxel.

chemotherapeutic treatments. The factors that make a specific tumor more or less sensitive to an anticancer drug, in this case paclitaxel, are largely unknown. Consequently, it is of crucial importance to find a way to identify genes that would aid in deciding in advance which patients should be candidates for treatment and which ones should not.

The use of RNAi in performing large scale, and even genome-wide, screens has become fairly well established. 
There are, however, few examples of RNAi screens using human cells and even fewer examples that utilize shRNA. We have shown that shRNA can be used in a screen that seeks to identify factors influencing drug sensitivity in human cell lines.

In establishing a proof-of-concept for this screen we chose to knockdown $M D R 1$, a gene that has a well-documented role in drug sensitivity (reviewed in 16). The protein it encodes for, $\mathrm{P}$-glycoprotein (P-gp, has an unusually long half-life, thus the utilization of shRNAs to convey stable and long-term knockdown was essential to the success of the screen. MDRI has been shown to play a role in response to a wide variety of chemotherapies, including paclitaxel sensitivity in clinical studies of patients with NSCLC (17) as well as in NSCLC cell lines (18). We demonstrate here that in A549 cell lines the level of MDRl is directly related to the level of response those cells have to paclitaxel at a fixed concentration. While previous reports have noted the correlation between drug sensitivity and $M D R 1$, we chose to directly target the expression of the gene at the transcript level. This has been accomplished in other cell lines using siRNA (19) and antisense oligonucleotides (20), yet this is the first example to our knowledge of using shRNA to increase drug sensitivity through MDRl modulation in a lung cancer cell line.

By screening a panel of tumor suppressor shRNAs, we found that the down-regulation of a number of genes significantly increases the level of resistance A549 cells have towards paclitaxel. This is not a surprising result, as the loss of tumor suppressor genes have been documented to play a role in drug sensitivity. We found, for example, that the knockdown of PTEN caused cells to have an increased resistance to paclitaxel by two- to four-fold. The actual role of PTEN in sensitivity to antineoplastic agents has been investigated in depth with initial studies showing that paclitaxel causes an up-regulation of the protein (21). More recent studies have shown that by decreasing levels of PTEN through modulation of $\mathrm{NF} K \mathrm{~B}$, cells can be made to be more resistant to paclitaxel (22) and PTEN negative cell lines have been shown to be more resistant to paclitaxel than PTEN positive cell lines (13).

Perhaps the more exciting results were those showing that a decrease in levels of certain tumor suppressors may result in an increased sensitivity to a chemotherapeutic agent. While this study is the first, to our knowledge, showing that lung cancer cells can be sensitized to paclitaxel through the loss of tumor suppressors, it is not the first to suggest that decreased levels of tumor suppressors result in increased drug sensitivity. It has been shown in MCF-7 (breast cancer) and SKOV3 (ovarian cancer) cells that inhibition of BRCA1, a known tumor suppressor, leads to increased sensitivity to cisplatin (14). Numerous clinical retrospective studies have confirmed this correlation between BRCA 1 and drug sensitivity, including one that evaluated the response of lung cell carcinoma patients given dual gemcitabine/cisplatin chemotherapy (15).

Our screen revealed that by decreasing VHL levels through shRNA, A549 cells displayed an increased sensitivity to paclitaxel. We further examined the actual level of knockdown seen after infection with each of the $V H L$ shRNAs. Those clones that yielded the greatest knockdown of
VHL also led to the most increased sensitivity to the drug. In addition, a threshold effect can be seen in that the clone resulting in $\sim 40 \%$ remaining endogenous protein level failed to confer increased sensitivity, while those clones causing endogenous levels to fall to at least $25 \%$ caused substantial sensitivity. VHL deficiency has been noted to improve renal cell carcinoma patient response to anti-VEGF therapy (23) and VHL has been seen to be a general prognostic marker for patients with RCC (24). No previous work has noted a correlation between VHL in NSCLC and chemotherapy response.

The results of this screen offer considerable prospects for the future development of biomarkers or therapeutic targets. Each of the tumor suppressors found to correlate with a differential response to paclitaxel is a potential biomarker for a response to this drug. Retrospective analysis of patients treated with paclitaxel should be performed in order to assess the correlation between the expression of these genes and clinical outcome. In addition, in vivo validation of these genes will give additional biological support to the role that they may play in paclitaxel sensitivity. Lentiviral delivery of shRNA, as used in this screen, makes future in vivo validation steps in the form of xenograft experiments straightforward and practical.

\section{References}

1. American Cancer Society. Cancer Facts and Figures, 2005. Available at http://www.cancer.org/docroot/stt/stt_0_2005.asp.

2. Clegg A, Scott DA, Sidhu M, Hewitson P and Waugh N: A rapid and systematic review of the clinical effectiveness and costeffectiveness of paclitaxel, docetaxel, gemcitabine and vinorelbine in non-small-cell lung cancer. Health Technol Assess 5: 1-195, 2001.

3. Gatzemeier U, Heckmayr M, Neuhauss R, Schluter I, Pawel JV, Wagner H and Dreps A: Phase II study with paclitaxel for the treatment of advanced inoperable non-small cell lung cancer. Lung Cancer 12: S101-S106, 1995.

4. Hainsworth JD, Thompson DS and Greco FA: Paclitaxel by 1-hour infusion: an active drug in metastatic non-small-cell lung cancer. J Clin Oncol 13: 1609-1614, 1995.

5. Pirker R, Krajnik G, Zochbauer S, Malayeri R, Kneussl M and Huber H: Paclitaxel/cisplatin in advanced non-small-cell lung cancer (NSCLC). Ann Oncol 6: 833-835, 1995.

6. Bonomi P: Review of paclitaxel/carboplatin in advanced nonsmall cell lung cancer. Semin Oncol 26: 55-59, 1999.

7. Akerley W and Choy H: Single-agent paclitaxel and radiation for non-small cell lung cancer. Semin Radiat Oncol 9: 85-89, 1999.

8. van de Vijver MJ, He YD, van't Veer LJ, Dai H, Hart AA, Voskuil DW, Schreiber GJ, Peterse JL, Roberts C, Marton MJ, Parrish M, Atsma D, Witteveen A, Glas A, Delahaye L, van der Velde T, Bartelink H, Rodenhuis S, Rutgers ET, Friend SH, and Bernards R: A gene-expression signature as a predictor of survival in breast cancer. N Engl J Med 347: 1999-2009, 2002.

9. van 't Veer LJ, Dai H, van de Vijver MJ, He YD, Hart AA, Mao M, Peterse HL, van der Kooy K, Marton MJ, Witteveen AT, Schreiber GJ, Kerkhoven RM, Roberts C, Linsley PS, Bernards R and Friend SH: Gene expression profiling predicts clinical outcome of breast cancer. Nature 415: 530-536, 2002.

10. MacKeigan JP, Murphy LO and Blenis J: Sensitized RNAi screen of human kinases and phosphatases identifies new regulators of apoptosis and chemoresistance. Nat Cell Biol 7: 591-600, 2005.

11. Aza-Blanc P, Cooper CL, Wagner K, Batalov S, Deveraux QL and Cooke MP: Identification of modulators of TRAIL-induced apoptosis via RNAi-based phenotypic screening. Mol Cell 12: 627-637, 2003

12. Scagliotti GV, Novello $S$ and Selvaggi G: Multidrug resistance in non-small-cell lung cancer. Ann Oncol 10: S83-S86, 1999.

13. Lee JT Jr, Steelman LS and McCubrey JA: Phosphatidylinositol 3'-kinase activation leads to multidrug resistance protein-1 expression and subsequent chemoresistance in advanced prostate cancer cells. Cancer Res 64: 8397-8404, 2004. 
14. Husain A, He G, Venkatraman ES and Spriggs DR: BRCA1 upregulation is associated with repair-mediated resistance to cisdiamminedichloroplatinum (II). Cancer Res 58: 1120-1123, 1998.

15. Taron M, Rosell R, Felip E, Mendez P, Souglakos J, Ronco MS, Queralt C, Majo J, Sanchez JM, Sanchez JJ and Maestre J: BRCA1 mRNA expression levels as an indicator of chemoresistance in lung cancer. Hum Mol Genet 13: 2443-2449, 2004.

16. Huang Y and Sadee W: Membrane transporters and channels in chemoresistance and -sensitivity of tumor cells. Cancer Lett 239: 168-182, 2005.

17. Yeh JJ, Hsu WH, Wang JJ, Ho ST and Kao A: Predicting chemotherapy response to paclitaxel-based therapy in advanced non-small-cell lung cancer with P-glycoprotein expression. Respiration 70: 32-35, 2003.

18. Pesic M, Markovic JZ, Jankovic D, Kanazir S, Markovic ID, Rakic L and Ruzdijic S: Induced resistance in the human non small cell lung carcinoma (NCI-H460) cell line in vitro by anticancer drugs. J Chemother 18: 66-73, 2006.

19. Wu H, Hait WN and Yang JM: Small interfering RNA-induced suppression of MDR1 (P-glycoprotein) restores sensitivity to multidrug-resistant cancer cells. Cancer Res 63: 1515-1519, 2003.
20. Chen L and Liu Y: Reversal of multidrug resistance in lung adenocarcinoma-resistant cell line A549/R by mdr1 antisense oligodeoxynucleotides in vitro. Zhonghua Yi Xue Za Zhi 80: 219-221, 2000.

21. Schondorf T, Becker M, Gohring UJ, Wappenschmidt B, Kolhagen $\mathrm{H}$ and Kurbacher CM: Interaction of cisplatin, paclitaxel and adriamycin with the tumor suppressor PTEN. Anticancer Drugs 12: 797-800, 2001.

22. Xia D, Srinivas H, Ahn YH, Sethi G, Sheng X, Yung WK, Xia Q, Chiao PJ, Kim H, Brown PH, Wistuba II, Aggarwal B and Kurie JM: Mitogen-activated protein kinase kinase-4 promotes cell survival by decreasing PTEN expression through an NFKBdependent pathway. J Biol Chem 282: 3507-3519, 2007.

23. Rini BI, Jaeger E, Weinberg V, Sein N, Chew K, Fong K, Simko J, Small EJ and Waldman FM: Clinical response to therapy targeted at vascular endothelial growth factor in metastatic renal cell carcinoma: impact of patient characteristics and Von Hippel-Lindau gene status. BJU Int 4: 756-762, 2006.

24. Yao M, Yoshida M, Kishida T, Nakaigawa N, Baba M, Kobayashi K, Miura T, Moriyama M, Nagashima Y, Nakatani Y, Kubota Y and Kondo K: VHL tumor suppressor gene alterations associated with good prognosis in sporadic clear-cell renal carcinoma. J Natl Cancer Inst 94: 1569-1575, 2002. 